This is a postprint version of the following published document:

Huertas, E.J., Marcellán, F., \& Pérez-Valero, M.F.

(2014). Asymptotics for Laguerre-Sobolev type ortogonal polynomials modified within their oscillatory regime. Applied Mathematics and Computation, 236, pp. 260-272.

DOI: $10.1016 /$ j.amc.2014.03.03

(C) Elsevier 2014

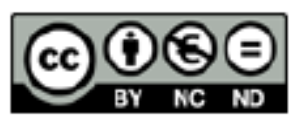

This work is licensed under a Creative Commons Attribution-NonCommercialNoDerivatives 4.0 International License. 


\title{
Asymptotics for Laguerre-Sobolev type orthogonal polynomials modified within their oscillatory regime
}

\author{
Edmundo J. Huertas ${ }^{\mathrm{a}, *, 1}$, Francisco Marcellán ${ }^{\mathrm{b}, 2}$, M. Francisca Pérez-Valero ${ }^{\mathrm{b}, 3}$, \\ Yamilet Quintanac,4
}

${ }^{a}$ CMUC, Departamento de Matemática, FCTUC, Universidade de Coimbra, Largo D. Dinis, Apdo. 3008, 3001-544 Coimbra, Portugal

${ }^{\mathrm{b}}$ Departamento de Matemáticas, Universidad Carlos III de Madrid, 28911 Leganés, Madrid, Spain

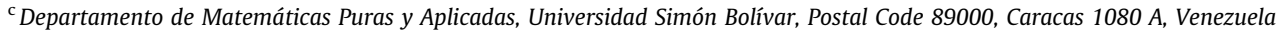

\section{A B S T R A C T}

In this paper we consider sequences of polynomials orthogonal with respect to the discrete Sobolev inner product

$$
\langle f, g\rangle_{S}=\int_{0}^{\infty} f(x) g(x) x^{\alpha} e^{x} d x+\mathbb{F}(c) A G(c)^{t}, \quad \alpha>1,
$$

where $f$ and $g$ are polynomials with real coefficients, $A \in \mathbb{R}^{(2,2)}$ and the vectors $\mathbb{F}(c), \mathbb{G}(c)$ are

$$
A=\left(\begin{array}{cc}
M & 0 \\
0 & N
\end{array}\right), \quad \mathbb{F}(c)=\left(f(c), f^{\prime}(c)\right) \quad \text { and } \mathbb{G}(c)=\left(g(c), g^{\prime}(c)\right), \text { respectively, }
$$

with $M, N \in \mathbb{R}_{+}$and the mass point $c$ is located inside the oscillatory region for the classical Laguerre polynomials. We focus our attention on the representation of these polynomials in terms of classical Laguerre polynomials and we analyze the behavior of the coefficients of the corresponding five term recurrence relation when the degree of the polynomials is large enough. Also, the outer relative asymptotics of the Laguerre Sobolev type with re spect to the Laguerre polynomials is analyzed.

Keywords: Orthogonal polynomials, Discrete Sobolev polynomials, Laguerre polynomials, Asymptotics.

\section{Introduction}

The study of asymptotic properties for general orthogonal polynomials is an important challenge in approximation theory and their applications permeate many fields in science and engineering [30,32,40,41]. Although it may seem as an old subject from the point of view of standard orthogonality [5,41], this is not the case neither in the general setting (cf. [16,17,30,

\footnotetext{
* Corresponding author.

E-mail address: ehuertas@mat.uc.pt (E.J. Huertas).

1 Partially supported by Dirección General de Investigación Científica y Técnica, Ministerio de Economía y Competitividad of Spain, grant MTM 2012-36732C03-01, and Fundacão para a Ciência e Tecnologia (FCT) of Portugal, ref. SFRH/BPD/91841/2012.

2 Supported by Dirección General de Investigación Científica, Ministerio de Economía y Competitividad of Spain, grant MTM $2012-36732-C 03-01$.

3 Supported by the Research Fellowship Program, Ministerio de Ciencia e Innovación (MTM 2009-12740-C03-01) and Dirección General de Investigación Científica, Ministerio de Economía y Competitividad of Spain, grant MTM 2012-36732-C03-01.

4 Partially supported by Dirección General de Investigación Científica, Ministerio de Economía y Competitividad of Spain, grant MTM 2012-36732-C03-01.
} 
3638,40 ]) nor from the viewpoint of Sobolev orthogonality, where it remains like a partially explored subject [3]. In fact, in the last ten years this topic has attracted the interest of many researchers [4,7 10,12,13,19,22 24,26,33 35].

A Sobolev type or discrete Sobolev type inner product on the linear space $\mathbb{P}$ of polynomials with real coefficients is de fined by

$$
\langle f, g\rangle_{s} \quad \int f(x) g(x) d \mu_{0}(x)+\sum_{k=0}^{d} \mathbb{F}\left(c_{k}\right) A_{k} \mathbb{G}\left(c_{k}\right)^{t}, \quad d \in \mathbb{Z}_{+},
$$

where $\mu_{0}$ is a nontrivial finite and positive Borel measure supported on the real line, $f, g \in \mathbb{P}$, and for $k \quad 0, \ldots, d, d \in \mathbb{Z}_{+}$, the matrices $A_{k} \quad\left(a_{i j}^{(k)}\right) \in \mathbb{R}^{\left(1+N_{k}\right)\left(1+N_{k}\right)}$ are positive semi definite. We denote by $\mathbb{E}\left(c_{k}\right)$ and $\mathbb{G}\left(c_{k}\right)$ the vectors $\mathbb{F}\left(c_{k}\right)$ $\left(f\left(c_{k}\right), f^{\prime}\left(c_{k}\right), \ldots, f^{\left(N_{k}\right)}\left(c_{k}\right)\right)$ and $\mathbb{G}\left(c_{k}\right) \quad\left(g\left(c_{k}\right), g^{\prime}\left(c_{k}\right), \ldots, g^{\left(N_{k}\right)}\left(c_{k}\right)\right)$, respectively, with $c_{k} \in \mathbb{R}, N_{k} \in \mathbb{Z}_{+}$and, as usual, $v^{t}$ denotes the transpose of the vector $v$. This notion was initially introduced in [11] for diagonal matrices $A_{k}$ in order to study recurrence relations for sequences of polynomials orthogonal with respect to (1).

The study of asymptotic properties of the sequences of orthogonal polynomials with respect to particular cases of the inner product (1) has been done by considering separately the cases 'mass points inside' or 'mass points outside' of supp $\mu_{0}$, respectively, being supp $\mu_{0}$ a bounded interval of $\mathbb{R}$ or, more recently, an unbounded interval of the real line (see, for instance [7 10,12,19,26]). The first results in the literature about asymptotic properties of orthogonal polynomials with respect to a


with $\lambda>0$. Therein, such asymptotic properties when there is only one mass point supporting the derivatives either inside or outside [ 1,1$]$ and $\mu$ is a measure in the Nevai class $M(0,1)$ are studied.

In [17], using an approach based on the theory of Padé approximants, the authors obtain the outer relative asymptotics for orthogonal polynomials with respect to the Sobolev type inner product (1) assuming that $\mu_{0}$ belongs to Nevai class $M(0,1)$ and the mass points $c_{k}$ belong to $\mathbb{C} \backslash \operatorname{supp} \mu$. The same problem with the mass points in supp $\mu \quad[1,1]$ was solved in [39], provided that $\mu^{\prime}(x)>0$ a.e. $x \in[1,1]$ and $A_{k}$ being diagonal matrices with $a_{i i}^{(k)}$ non negative constants. The pointwise conver gence of the Fourier series associated to such an inner product was studied when $\mu_{0}$ is the Jacobi measure (see also [20,21]). On the other hand, the asymptotics for orthogonal polynomials with respect to the Sobolev type inner product (1) with $\mu_{0} \in M(0,1), c_{k}$ belong to supp $\mu \backslash[1,1]$, and $A_{k}$ are complex diagonal matrices such that $a_{1+N_{k}, 1+N_{k}}^{(k)} \neq 0$, was solved in [2].

Another results about the asymptotic behavior of orthogonal polynomials associated with diagonal (resp. non diagonal) Sobolev inner products with respect to measures supported on the complex plane can be found in $[1,4,7,28]$. On the other hand, results concerning asymptotics for extremal polynomials associated to non diagonal Sobolev norms may be seen in [29,33 35].

In this paper we deal with sequences of polynomials orthogonal with respect to a particular case of (1). Indeed, $\mu_{0}$ is the Laguerre classical measure

$$
\langle f, g\rangle_{S} \quad \int_{0}^{\infty} f(x) g(x) x^{\alpha} e^{x} d x+\mathbb{F}(c) A \mathbb{G}(c)^{t}, \quad \alpha>1,
$$

$f, g \in \mathbb{P}$. The matrix $A$ and the vectors $\mathbb{F}(c), \mathbb{G}(c)$ are

$$
A \quad\left(\begin{array}{cc}
M & 0 \\
0 & N
\end{array}\right), \quad \mathbb{F}(c) \quad\left(f(c), f^{\prime}(c)\right) \text { and } \mathbb{G}(c) \quad\left(g(c), g^{\prime}(c)\right), \text { respectively, }
$$

$M, N \in \mathbb{R}_{+}$, and the mass point $c$ is located inside the oscillatory region for the classical Laguerre polynomials, i.e., $c>0$. Fol lowing the methodology given in [7 10,19,26] we focus our attention on the representation of these polynomials in terms of the classical Laguerre polynomials. Their asymptotic behavior will be discussed.

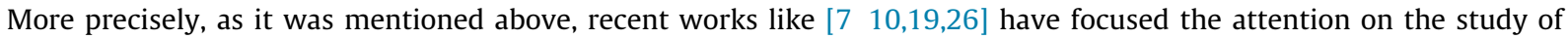
asymptotic properties of the sequences of orthogonal polynomials with respect to specific cases of the inner product (1) with 'mass points outside' of supp $\mu_{0}$, being supp $\mu_{0}$ an unbounded interval of the real line. However, to the best of our knowledge, asymptotic properties of the sequences of orthogonal polynomials associated to (2) are not available in the literature.

The structure of the manuscript is as follows. Section 2 contains the basic background about Laguerre polynomials and some other auxiliary results which will be used throughout the paper. In Section 3 we prove our main result, namely the outer relative asymptotic of the Laguerre Sobolev type orthogonal polynomials modified into the positive real semiaxis. Fi nally, in Section 4 we deduce the coefficients of the corresponding five term recurrence relation as well as their asymptotic behavior when the degree of the polynomials is large enough.

Throughout this manuscript, the notation $u_{n} \sim v_{n}$ means that the sequence $\left\{\frac{u_{n}}{v_{n}}\right\}_{n}$ converges to 1 as $n \rightarrow \infty$. Any other stan dard notation will be properly introduced whenever needed.

\section{Background and previous results}

Laguerre orthogonal polynomials are defined as the polynomials orthogonal with respect to the inner product

$$
\langle f, g\rangle_{\alpha} \quad \int_{0}^{\infty} f(x) g(x) x^{\alpha} e^{x} d x, \quad \alpha>1, \quad f, g \in \mathbb{P} .
$$


The expression of these polynomials as an ${ }_{1} F_{1}$ hypergeometric function is very well known in the literature (see for in stance, $[15,31,41])$. The connection between these two facts follows from a characterization of such orthogonal polynomials as eigenfunctions of a second order linear differential operator with polynomial coefficients. The following proposition will be useful in the sequel and it summarizes some structural and asymptotic properties of Laguerre polynomials involving two different normalizations $[5,18,41]$.

Proposition 2.1. Let $\left\{\widehat{L}_{n}^{\alpha}(x)\right\}_{n \geqslant 0}$ be the sequence of monic Laguerre orthogonal polynomials. Then the following statements hold.

(1) Three term recurrence relation. For every $n \geqslant 1$

$$
x \widehat{L}_{n}^{\alpha}(x) \quad \widehat{L}_{n+1}^{\alpha}(x)+\beta_{n} \widehat{L}_{n}^{\alpha}(x)+\gamma_{n} \widehat{L}_{n}^{\alpha}{ }_{1}(x)
$$

with initial conditions $\widehat{L}_{0}^{\alpha}(x) \quad 1, \widehat{L}_{1}^{\alpha}(x) \quad x \quad(\alpha+1)$, and $\beta_{n} \quad 2 n+\alpha+1, \gamma_{n} \quad n(n+\alpha)$.

(2) For every $n \in \mathbb{N}$,

$$
\left\|\widehat{L}_{n}^{\alpha}\right\|_{\alpha}^{2} \quad \Gamma(n+1) \Gamma(n+\alpha+1) .
$$

(3) Hahn's condition. For every $n \in \mathbb{N}$,

$$
\left[\widehat{L}_{n}^{\alpha}(x)\right]^{\prime} \quad n \widehat{L}_{n 1}^{\alpha+1}(x) .
$$

(4) The nth Dirichlet kernel $K_{n}(x, y)$, given by

$$
K_{n}(x, y) \quad \sum_{k 0}^{n} \frac{\widehat{L}_{k}^{\alpha}(x) \widehat{L}_{k}^{\alpha}(y)}{\left\|\widehat{L}_{k}^{\alpha}\right\|_{\alpha}^{2}}
$$

satisfies the Christoffel Darboux formula (cf. [41] [Theorem 3.2.2]):

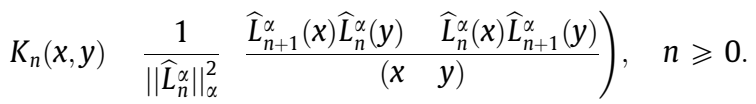

(5) The so called confluent form of the above kernel is given by

$$
K_{n}(x, x) \quad \frac{1}{\left\|\widehat{L}_{n}^{\alpha}\right\|_{\alpha}^{2}}\left\{\left[\widehat{L}_{n+1}^{\alpha}\right]^{\prime}(x) \widehat{L}_{n}^{\alpha}(x) \quad\left[\widehat{L}_{n}^{\alpha}\right]^{\prime}(x) \widehat{L}_{n+1}^{\alpha}(x)\right\}, \quad n \geqslant 0 .
$$

(6) Let $\left\{L_{n}^{(\alpha)}(x)\right\}_{n \geqslant 0}$ be the sequence of Laguerre orthogonal polynomials with leading coefficient $\frac{(1)^{n}}{n !}$, then

$$
L_{n}^{(\alpha)}(x) \quad \frac{(1)^{n}}{n !} \widehat{L}_{n}^{\alpha}(x)
$$

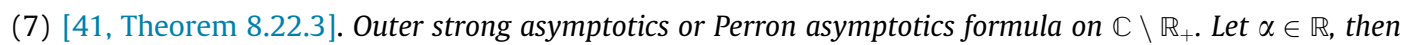

$$
L_{n}^{(\alpha)}(x) \quad \frac{1}{2} \pi^{1 / 2} e^{x / 2}(x)^{\alpha / 2}{ }^{1 / 4} n^{\alpha / 2} 1^{1 / 4} \exp \left(2(n x)^{1 / 2}\right) \times\left\{\sum_{k=0}^{p} C_{k}(\alpha ; x) n^{k / 2}+\mathcal{O}\left(n^{p / 2}\right)\right\} .
$$

Here $C_{k}(\alpha ; x)$ is independent of $n$. This relation holds for $x$ in the complex plane with a cut along the positive real semiaxis, and it also holds if $x$ is in the cut plane mentioned. $(x)^{\alpha / 2}{ }^{1 / 4}$ and $(x)^{1 / 2}$ must be taken real and positive if $x<0$. The bound for the remainder holds uniformly in every compact subset of the complex plane with empty intersection with $\mathbb{R}_{+}$.

(8) [41, Theorem 8.22.2]. Perron generalization of Fejér formula on $\mathbb{R}_{+}$. Let $\alpha \in \mathbb{R}$. Then for $x>0$ we have

$$
\begin{aligned}
& L_{n}^{(\alpha)}(x) \quad \pi^{1 / 2} e^{x / 2} x^{\alpha / 2}{ }^{1 / 4} n^{\alpha / 2}{ }^{1 / 4} \cos \left\{2(n x)^{1 / 2} \quad \alpha \pi / 2 \quad \pi / 4\right\} \cdot\left\{\sum_{k}^{p} A_{k}(x) n^{k / 2}+\mathcal{O}\left(n^{p / 2}\right)\right\} \\
& +\pi^{1 / 2} e^{x / 2} x^{\alpha / 2}{ }^{1 / 4} n^{\alpha / 2}{ }^{1 / 4} \sin \left\{2(n x)^{1 / 2} \quad \alpha \pi / 2 \quad \pi / 4\right\} \cdot\left\{\sum_{k 0}^{p} B_{k}(x) n^{k / 2}+\mathcal{O}\left(n^{p / 2}\right)\right\},
\end{aligned}
$$

where $A_{k}(x)$ and $B_{k}(x)$ are certain functions of $x$ independent of $n$ and regular for $x>0$. The bound for the remainder holds uni formly in $[\epsilon, \omega]$. For $k \quad 0$ we have $A_{0}(x) \quad 1$ and $B_{0}(x) \quad 0$.

Next, we summarize some results about the so called $k$ iterated Christoffel perturbed Laguerre orthogonal polynomials. They are orthogonal with respect to the inner product

$$
\langle f, g\rangle_{[k]} \quad \int_{0}^{\infty} f(x) g(x)(x \quad c)^{k} x^{\alpha} e^{x} d x, \quad \alpha>1, \quad f, g \in \mathbb{P},
$$


and we will denote by $\left\{\widehat{L}_{n}^{\alpha, k]}(x)\right\}_{n \geqslant 0}$ the corresponding monic sequence and by

$$
\left\|\widehat{L}_{n}^{\alpha,[k]}\right\|_{[k]}^{2} \quad\left\langle\widehat{L}_{n}^{\alpha,[k]}(x), x^{n}\right\rangle_{[k]}
$$

the norm of the $n$th degree polynomial in the sequence. Note that the modified Laguerre measure $(x \quad c)^{k} x^{\alpha} e^{x} d x$ is positive definite when either $k$ is an even integer or $k$ is an odd number and $c$ is outside the interval $[0,+\infty)$. It is very well known that, when $k \quad 1$ and $c$ is outside the support of the classical Laguerre measure, i.e., when it is assumed that $c$ is not a zero of $\widehat{L}_{n}^{\alpha}(x)$, these polynomials are actually the monic Laguerre kernels (8) (see [5], [Sec. I.7]).

We introduce the following standard notation for the partial derivatives of the $n$th Dirichlet kernel $K_{n}(x, y)$

$$
\frac{\partial^{j+k} K_{n}(x, y)}{\partial x^{j} \partial y^{k}} \quad K_{n}^{(j, k)}(x, y), \quad 0 \leqslant i, j \leqslant n .
$$

Taking derivatives with respect to $y$ in (7) and considering $x \quad y \quad c$ we get

$$
K_{n 1}^{(0,1)}(c, c) \quad \frac{1}{2} \frac{\widehat{L}_{n 1}^{\alpha}(c)\left[\widehat{L}_{n}^{\alpha}\right]^{\prime \prime}(c) \widehat{L}_{n}^{\alpha}(c)\left[\widehat{L}_{n 1}^{\alpha}\right]^{\prime \prime}(c)}{\Gamma(n) \Gamma(n+\alpha)} .
$$

On the other hand,

$$
K_{n 1}^{(1,1)}(c, c) \quad \frac{1}{3 !} \frac{1}{\Gamma(n) \Gamma(n+\alpha)} \cdot\left\{\widehat{L}_{n}^{\alpha}{ }_{1}(c)\left[\widehat{L}_{n}^{\alpha}\right]^{\prime \prime \prime}(c)+3\left[\widehat{L}_{n}^{\alpha}\right]_{1}^{\prime}(c)\left[\widehat{L}_{n}^{\alpha}\right]^{\prime \prime}(c) \quad \widehat{L}_{n}^{\alpha}(c)\left[\widehat{L}_{n}^{\alpha}\right]^{\prime \prime \prime}(c) \quad 3\left[\widehat{L}_{n}^{\alpha}\right]^{\prime}(c)\left[\widehat{L}_{n}^{\alpha}\right]^{\prime \prime}(c)\right\} .
$$

Remark 2.1. The local character of the Taylor expansions means (14) and (15) hold for every $c \in \mathbb{R}$. However, we are only interested in the case $c>0$ in order to study the asymptotic behavior of sequences of polynomials orthogonal with respect to the Sobolev type inner product (2).

The first technical step required for the proof of our main result is the following lemma, concerning the asymptotic behav ior as $n \rightarrow \infty$ of the above Laguerre kernels at $x \quad c, c \in \mathbb{R}_{+}$, that is, within the oscillatory regime of the classical Laguerre orthogonal polynomials.

Lemma 2.1. For every $c>0$, we have

$$
\begin{aligned}
& K_{n 1}(c, c) \sim \pi^{1} e^{c} c^{\frac{1}{2} \alpha} n^{1 / 2}, \\
& K_{n 1}^{(0,1)}(c, c) \sim \pi^{1} e^{c} c^{\frac{1}{2} \alpha} n^{1 / 2}, \\
& K_{n 1}^{(1,1)}(c, c) \sim \frac{1}{3} \pi^{1} e^{c} c^{\frac{3}{2} \alpha} n^{3 / 2} .
\end{aligned}
$$

Proof. Taking $p \quad 1$ in (12), we have $A_{0}(x) \quad 1$ and $B_{0}(x) \quad 0$. Thus, we obtain the behavior of $\widehat{L}_{n}^{(\alpha)}(x)$ for $n$ large enough, when $x \in \mathbb{R}_{+}$

$$
\widehat{L}_{n}^{\alpha}(x) \quad(1)^{n} \Gamma(n+1) \pi^{1 / 2} e^{x / 2} x^{\alpha / 2}{ }^{1 / 4} n^{\alpha / 2}{ }^{1 / 4} \cdot \cos \left\{2(n x)^{1 / 2} \quad \alpha \pi / 2 \quad \pi / 4\right\} \cdot\left(1+\mathcal{O}\left(n^{1 / 2}\right)\right) .
$$

We can rewrite the above expression as

$$
\widehat{L}_{n}^{\alpha}(x) \quad(1)^{n} \Gamma(n+1) n^{\frac{\alpha}{2} \frac{1}{4}} \sigma^{\alpha}(x) \cos \varphi_{n}^{\alpha}(x)\left(1+\mathcal{O}\left(n^{1 / 2}\right)\right)
$$

where

$$
\varphi_{n}^{\alpha}(x) \quad 2(n x)^{1 / 2} \quad \frac{\alpha \pi}{2} \quad \frac{\pi}{4}
$$

and

$$
\sigma^{\alpha}(x) \quad \pi^{1 / 2} e^{x / 2} x^{\alpha / 2} \quad 1 / 4
$$

being a function independent of $n$. Combining (6) with (16), we get

$$
K_{n}{ }_{1}(c, c) \sim \frac{\Gamma(n+1)}{\Gamma(n+\alpha)} n^{\alpha} \Theta_{n}(c ; \alpha),
$$

where

$$
\Theta_{n}(c ; \alpha) \quad \sigma^{\alpha}(c) \sigma^{\alpha+1}(c)\left[\cos \varphi_{n}^{\alpha+1}(c) \cos \varphi_{n}^{\alpha}(c) \quad \cos \varphi_{n}^{\alpha+1}(c) \cos \varphi_{n}^{\alpha}(c)\right] .
$$


Let us examine the above expression. Using the trigonometric identity

$$
\cos (a) \cos (b) \quad \frac{\cos (a+b)+\cos (a \quad b)}{2},
$$

we have

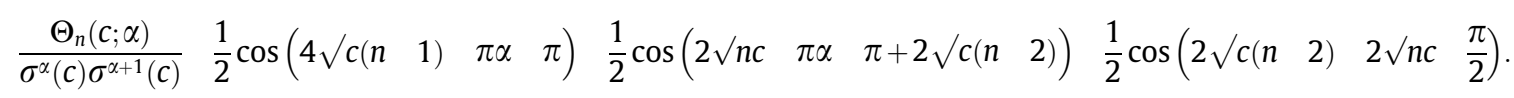

The last term on the right hand side is

$$
\frac{1}{2} \cos \left(\begin{array}{lllll}
2 \sqrt{ }\left(\begin{array}{llll}
n & 2) c & 2 \sqrt{ } n c & \frac{\pi}{2}
\end{array}\right) \quad \frac{1}{2} \sin \left(\begin{array}{lll}
2 \sqrt{ } n c & 2 \sqrt{ }(n & 2
\end{array}\right) c
\end{array}\right)
$$

which behaves with $n$ as follows

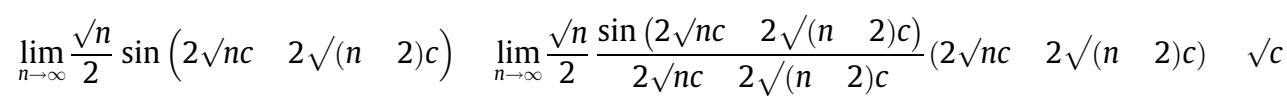

and, therefore,

$$
\frac{1}{2} \sin \left(2 \sqrt{ }\left(\begin{array}{lll}
n & 2) c & 2 \sqrt{ } n c
\end{array}\right) \sim \sqrt{ } \frac{c}{n} .\right.
$$

Next we study

$$
\left.\frac{1}{2} \cos \left(\begin{array}{llll}
4 \sqrt{ } c(n & 1
\end{array}\right) \quad \pi \alpha \quad \pi\right) \quad \frac{1}{2} \cos (2 \sqrt{ } n c \quad \pi \alpha \quad \pi+2 \sqrt{ } c(n \quad 2))
$$

in (19). Using

$$
\cos a \cos b \quad 2 \sin \left(\frac{a+b}{2}\right) \sin \left(\frac{a \quad b}{2}\right),
$$

we get that (21) becomes

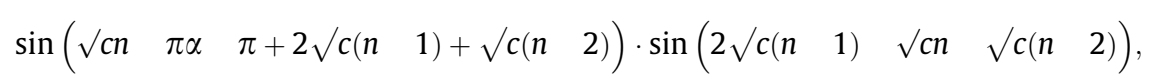

where the first factor is bounded, and the second verifies

$$
\lim _{n \rightarrow \infty} \sqrt{ } n \sin \left(2 \sqrt{ } c\left(\begin{array}{lllll}
n & 1
\end{array}\right) \quad \sqrt{ } c n \quad \sqrt{ } c\left(\begin{array}{ll}
n & 2
\end{array}\right)\right) \quad 0 .
$$

From (20) and (22), we conclude

$$
\Theta_{n}(c ; \alpha) \sim \pi{ }^{1} e^{c} c^{\frac{1}{2} \alpha} n^{1 / 2} .
$$

On the other hand, from the Stirling's formula for the Gamma function, we deduce

$$
\frac{\Gamma(n+1)}{\Gamma(n+\alpha)} \sim n^{1} \alpha,
$$

under the above assumptions we get

$$
K_{n}{ }_{1}(c, c) \sim \pi^{1} e^{c} c^{\frac{1}{2} \alpha} n^{1 / 2}, \quad c \in \mathbb{R}_{+} .
$$

Next, we can proceed as above and we obtain the asymptotic behavior given in (14). For $n$ large enough, we get

$$
K_{n 1}^{(0,1)}(c, c) \sim \frac{1}{2} \frac{\Gamma(n+1)}{\Gamma(n+\alpha)} n^{\alpha+\frac{1}{2}} \Psi_{n}(c ; \alpha),
$$

where

$$
\Psi_{n}(c ; \alpha) \quad \sigma^{\alpha}(c) \sigma^{\alpha+2}(c)\left[\cos \varphi_{n}^{\alpha}(c) \cos \varphi_{n 3}^{\alpha+2}(c) \quad \cos \varphi_{n}^{\alpha}(c) \cos \varphi_{n 2}^{\alpha+2}(c)\right] .
$$

The expression in square brackets can be rewritten as

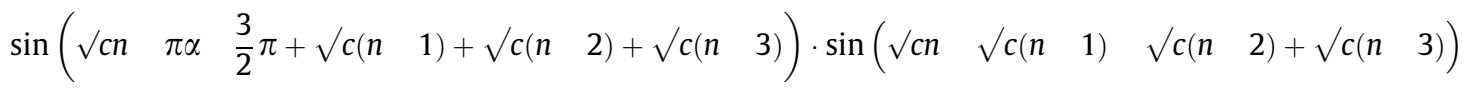

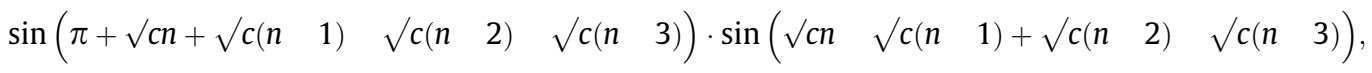


where

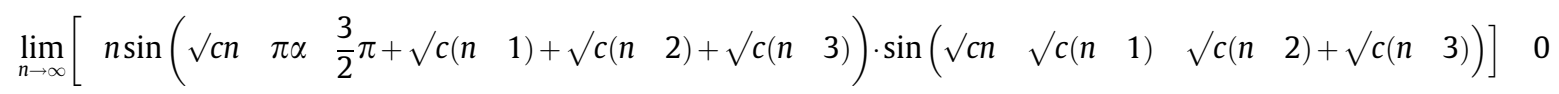

and

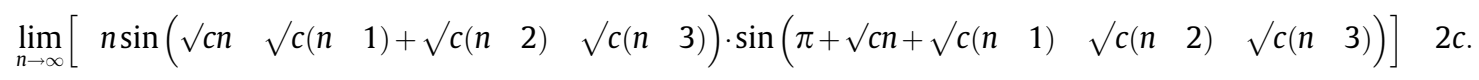

As a consequence, taking into account (17), we get

$$
\Psi_{n}(c ; \alpha) \sim \pi^{1} e^{c} c^{\alpha \frac{3}{2}} \cdot 2 c n^{1} .
$$

Replacing the above expression in (24), and using again (23), we conclude

$$
K_{n 1}^{(0,1)}(c, c) \sim \pi^{1} e^{c} c^{\frac{1}{2} \alpha} n^{1 / 2} .
$$

Finally,

$$
K_{n 1}^{(1,1)}(c, c) \sim \frac{\Gamma(n+1)}{\Gamma(n+\alpha)} n^{\alpha+1}\left(\frac{1}{3 !} \Lambda_{1, n}(c ; \alpha)+\frac{1}{2 !} \Lambda_{2, n}(c ; \alpha)\right),
$$

where

$$
\begin{array}{lll}
\Lambda_{1, n}(c ; \alpha) & \sigma^{\alpha}(c) \sigma^{\alpha+3}(c)\left[\cos \varphi_{n 3}^{\alpha+3}(c) \cos \varphi_{n}^{\alpha}{ }_{1}(c)\right. & \left.\cos \varphi_{n 4}^{\alpha+3}(c) \cos \varphi_{n}^{\alpha}(c)\right], \\
\Lambda_{2, n}(c ; \alpha) & \sigma^{\alpha+1}(c) \sigma^{\alpha+2}(c)\left[\cos \varphi_{n}^{\alpha+2}(c) \cos \varphi_{n 2}^{\alpha+1}(c)\right. & \left.\cos \varphi_{n 3}^{\alpha+2}(c) \cos \varphi_{n}^{\alpha+1}(c)\right] .
\end{array}
$$

The two expressions in square brackets of (26) and (27) can be rewritten respectively, as follows



$$
\begin{aligned}
& \left.\left.\sin \left(\begin{array}{llllll}
\sqrt{ } c(n & 3
\end{array}\right) \quad \sqrt{ } n c \quad \sqrt{ } c\left(\begin{array}{ll}
n & 1
\end{array}\right) \quad \frac{3}{2} \pi+\sqrt{ } c\left(\begin{array}{ll}
n & 4
\end{array}\right)\right) \cdot \sin \left(\begin{array}{lllll}
\sqrt{ } n c & \sqrt{ } c\left(\begin{array}{lll}
n & 1)+\sqrt{ } c(n & 3
\end{array}\right) & \sqrt{ } c(n & 4
\end{array}\right)\right),
\end{aligned}
$$



$$
\begin{aligned}
& \frac{1}{2} \cos \left(2 \sqrt{ } c(n+3) \quad 2 \sqrt{ } c(n+1) \quad \frac{1}{2} \pi\right),
\end{aligned}
$$

where the terms of each sumand in the above expressions have the following behavior

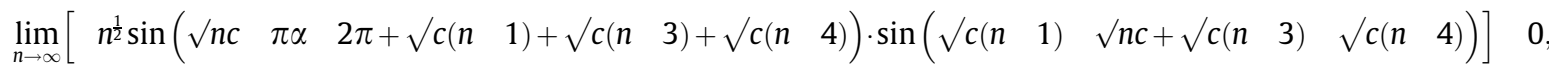

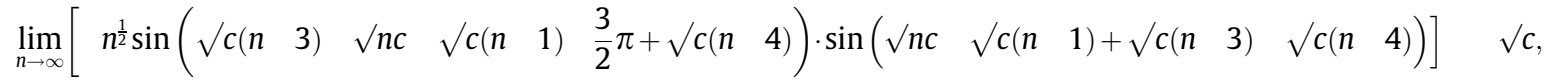

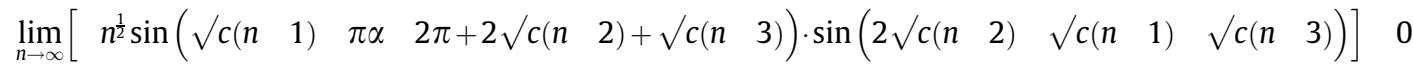

and

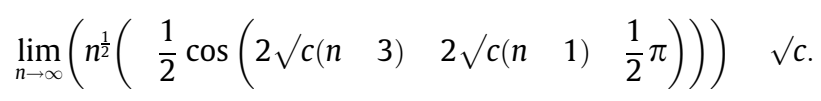

Hence, using again (17), we have

$$
\begin{aligned}
& \Lambda_{1, n}(c ; \alpha) \sim \pi^{1} e^{c} c^{2} \alpha \sqrt{ } c n^{1 / 2}, \\
& \Lambda_{2, n}(c ; \alpha) \sim \pi^{1} e^{c} c^{2} \alpha \sqrt{ } c n^{1 / 2} .
\end{aligned}
$$

Therefore

$$
\left(\frac{1}{3 !} \Lambda_{1, n}(c ; \alpha)+\frac{1}{2 !} \Lambda_{2, n}(c ; \alpha)\right) \sim \frac{1}{3} \pi^{1} e^{c} c^{\alpha \frac{3}{2}} n^{1 / 2} .
$$

Replacing in (25) we conclude,

$$
K_{n 1}^{(1,1)}(c, c) \sim \frac{1}{3} \pi{ }^{1} e^{c} c^{\frac{3}{2} \alpha} n^{3 / 2} .
$$




\section{Outer relative asymptotics for $c$ on $\boldsymbol{R}_{+}$}

The main result of this section will be the outer relative asymptotics for the Laguerre Sobolev type polynomials $\widehat{S}_{n}^{M, N}(x)$, orthogonal with respect to (2), when $c \in \mathbb{R}_{+}$. The proof will naturally fall in several parts, which will be established through an appropriate sequence of Lemmas.

First, we will present a well known expansion of the monic polynomials $\widehat{S}_{n}^{M, N}(x)$ in terms of classical Laguerre polynomials $\widehat{L}_{n}^{\alpha}(x)$. The most common way to represent the Laguerre Sobolev type orthogonal polynomials $\widehat{S}_{n}^{M, N}(x)$ is using the Laguerre kernel and its derivatives as follows (see [25] and Theorem 5.1 in [14]).

$$
\left(\begin{array}{lll}
x & c
\end{array}\right)^{2} \widehat{S}_{n}^{M, N}(x) \quad A(n ; x) \widehat{L}_{n}^{\alpha}(x)+B(n ; x) \widehat{L}_{n}^{\alpha}{ }_{1}(x),
$$

where

$$
\begin{array}{ll}
A(n ; x) & (x \quad c)^{2}+(x \quad c) A_{1}(n ; c)+A_{0}(n ; c) \\
B(n ; x) & (x \quad c) B_{1}(n ; c)+B_{0}(n ; c)
\end{array}
$$

with

$$
\begin{array}{ll}
A_{1}(n ; c) & \frac{M \widehat{S}_{n}^{M, N}(c) \widehat{L}_{n 1}^{\alpha}(c)}{\left\|\widehat{L}_{n 1}^{\alpha}\right\|_{\alpha}^{2}} \frac{N\left[\widehat{S}_{n}^{M, N}\right]^{\prime}(c)\left[\widehat{L}_{n}^{\alpha}\right]_{1}^{\prime}(c)}{\left\|\widehat{L}_{n}^{\alpha}\right\|_{\alpha}^{2}}, \\
A_{0}(n ; c) & \frac{N\left[\widehat{S}_{n}^{M, N}\right]^{\prime}(c) \widehat{L}_{n 1}^{\alpha}(c)}{\left\|\widehat{L}_{n}^{\alpha}\right\|_{1} \|_{\alpha}^{2}}, \\
B_{1}(n ; c) & \frac{M \widehat{S}_{n}^{M, N}(c) \widehat{L}_{n}^{\alpha}(c)}{\left\|\widehat{L}_{n}^{\alpha}\right\|_{\alpha}^{2}}+\frac{N\left[\widehat{S}_{n}^{M, N}\right]^{\prime}(c)\left[\widehat{L}_{n}^{\alpha}\right]^{\prime}(c)}{\left\|\widehat{L}_{n 1}^{\alpha}\right\|_{\alpha}^{2}}, \\
B_{0}(n ; c) & \frac{N\left[\widehat{S}_{n}^{M, N}\right]^{\prime}(c) \widehat{L}_{n}^{\alpha}(c)}{\left\|\widehat{L}_{n}^{\alpha}\right\|_{\alpha}^{2}} .
\end{array}
$$

Notice that

$$
\begin{aligned}
& \widehat{S}_{n}^{M, N}(c) \frac{\left|\begin{array}{cc}
\widehat{L}_{n}^{\alpha}(c) & N K_{n}^{(0,1)}(c, c) \\
{\left[\widehat{L}_{n}^{\alpha}\right]^{\prime}(c)} & 1+N K_{n}^{(1,1)}(c, c)
\end{array}\right|}{\left|\begin{array}{cc}
1+M K_{n}(c, c) & N K_{n 1}^{(0,1)}(c, c) \\
M K_{n}^{(1,0)}(c, c) & 1+N K_{n}^{(1,1)}(c, c)
\end{array}\right|},
\end{aligned}
$$

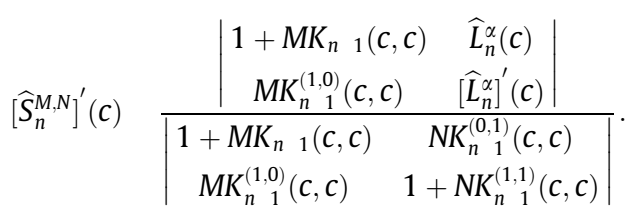

We will analyze the polynomial coefficients in the above expansion in order to obtain the desired results. If we replace (31) and (32) in (30), we obtain

$$
\begin{aligned}
& A_{1}(n ; c) \frac{M \widehat{L}_{n}^{\alpha}{ }_{1}(c) \widehat{L}_{n}^{\alpha}(c)}{M N \widehat{L}_{n 1}^{\alpha}(c) \widehat{L}_{n}^{\alpha}(c) K_{n}^{(1,1)}(c, c)+M N n \widehat{L}_{n 1}^{\alpha}(c) \widehat{L}_{n}^{\alpha+1}(c) K_{n}^{(0,1)}(c, c)} \\
& +\frac{\left(N n^{2} \widehat{L}_{n=2}^{\alpha+1}(c) \widehat{L}_{n 1}^{\alpha+1}(c) \quad M N n^{2} \widehat{L}_{n 2}^{\alpha+1}(c) \widehat{L}_{n 1}^{\alpha+1}(c) K_{n 1}(c, c)+M N n \widehat{L}_{n 2}^{\alpha+1}(c) \widehat{L}_{n}^{\alpha}(c) K_{n 1}^{(1,0)}(c, c)\right)}{\left\|\widehat{L}_{n 1}^{\alpha}\right\|_{\alpha}^{2}\left(1+M K_{n 1}(c, c)+N K_{n 1}^{(1,1)}(c, c)+M N K_{n 1}(c, c) K_{n 1}^{(1,1)}(c, c) \quad M N K_{n 1}^{(0,1)}(c, c) K_{n 1}^{(1,0)}(c, c)\right)},
\end{aligned}
$$

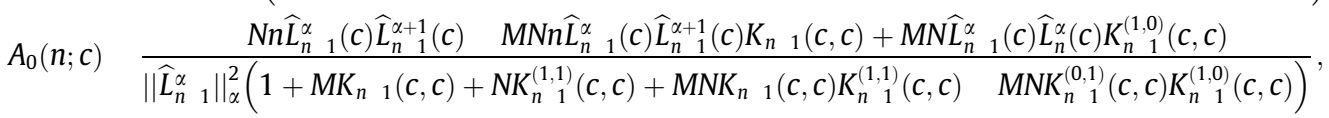

$$
\begin{aligned}
& B_{1}(n ; c) \frac{M \widehat{L}_{n}^{\alpha}(c) \widehat{L}_{n}^{\alpha}(c)+M N \widehat{L}_{n}^{\alpha}(c) \widehat{L}_{n}^{\alpha}(c) K_{n 1}^{(1,1)}(c, c)}{M N \widehat{L}_{n} \widehat{L}_{n}^{\alpha}(c) \widehat{L}_{n}^{\alpha+1}(c) K_{n 1}^{(0,1)}(c, c)} \\
& +\frac{N n^{2} \widehat{L}_{n}^{\alpha+1}(c) \widehat{L}_{n}^{\alpha+1}(c)+M N n^{2} \widehat{L}_{n 1}^{\alpha+1}(c) \widehat{L}_{n 1}^{\alpha+1}(c) K_{n}{ }_{1}(c, c) \quad M N n \widehat{L}_{n 1}^{\alpha+1}(c) \widehat{L}_{n}^{\alpha}(c) K_{n 1}^{(1,0)}(c, c)}{\left\|\widehat{L}_{n}^{\alpha}\right\|_{\alpha}^{2}\left(1+M K_{n}(c, c)+N K_{n 1}^{(1,1)}(c, c)+M N K_{n}{ }_{1}(c, c) K_{n 1}^{(1,1)}(c, c) \quad M N K_{n 1}^{(0,1)}(c, c) K_{n 1}^{(1,0)}(c, c)\right)},
\end{aligned}
$$




Using (16) and the estimates in Lemma 2.1, we can compute the asymptotic behavior of the previous expressions as follows.

$$
\begin{aligned}
& A_{1}(n ; c) \sim \frac{1}{N c \sigma^{\alpha+1}(c) \sigma^{\alpha+3}(c)} n^{3 / 2} \cos \varphi_{n}^{\alpha}{ }_{1}(c) \cos \varphi_{n}^{\alpha}(c)+\cos \varphi_{n}^{\alpha}{ }_{1}(c) \cos \varphi_{n}^{\alpha}(c)+2 \sqrt{ } c n{ }^{1 / 2} \cos \varphi_{n}^{\alpha}{ }_{1}(c) \cos \varphi_{n}^{\alpha+1}(c) \\
& +\frac{1}{M \sigma^{\alpha}(c) \sigma^{\alpha}(c)} n^{1 / 2} \cos \varphi_{n 2}^{\alpha+1}(c) \cos \varphi_{n}^{\alpha+1}(c)+\cos \varphi_{n}^{\alpha+1}(c) \cos \varphi_{n}^{\alpha+1}(c)+2 n^{1 / 2} \cos \varphi_{n}^{\alpha+1}(c) \cos \varphi_{n}^{\alpha}(c), \\
& A_{0}(n ; c) \sim \frac{1}{M c \sigma^{\alpha}(c) \sigma^{\alpha+3}(c)} n^{1} \cos \varphi_{n}^{\alpha}{ }_{1}(c) \cos \varphi_{n}^{\alpha+1}(c) \quad c^{1 / 2} n^{1 / 2} \cos \varphi_{n 1}^{\alpha}(c) \cos \varphi_{n}^{\alpha+1}(c) \quad 2 n^{1} \cos \varphi_{n 1}^{\alpha}(c) \cos \varphi_{n}^{\alpha}(c), \\
& B_{1}(n ; c) \sim \frac{1}{N c \sigma^{\alpha+1}(c) \sigma^{\alpha+3}(c)} n^{1 / 2} \cos \varphi_{n}^{\alpha}(c) \cos \varphi_{n}^{\alpha}(c)+n \cos \varphi_{n}^{\alpha}(c) \cos \varphi_{n}^{\alpha}(c)+2 \sqrt{ } c n^{1 / 2} \cos \varphi_{n}^{\alpha}(c) \cos \varphi_{n}^{\alpha+1}(c) \\
& +\frac{1}{M \sigma^{\alpha}(c) \sigma^{\alpha}(c)} n^{1 / 2} \cos \varphi_{n 1}^{\alpha+1}(c) \cos \varphi_{n 1}^{\alpha+1}(c)+n \cos \varphi_{n 1}^{\alpha+1}(c) \cos \varphi_{n 1}^{\alpha+1}(c)+2 n^{1 / 2} \cos \varphi_{n 1}^{\alpha+1}(c) \cos \varphi_{n}^{\alpha}(c), \\
& B_{0}(n ; c) \sim \frac{1}{M c \sigma^{\alpha}(c) \sigma^{\alpha+3}(c)} \cos \varphi_{n}^{\alpha}(c) \cos \varphi_{n}^{\alpha+1}(c) \quad c^{1 / 2} n^{1 / 2} \cos \varphi_{n}^{\alpha}(c) \cos \varphi_{n}^{\alpha+1}(c) \quad 2 \cos \varphi_{n}^{\alpha}(c) \cos \varphi_{n}^{\alpha}(c) .
\end{aligned}
$$

Due to the oscillatory behavior of the cosines functions appearing in the preceding formulas, there are no real numbers $\beta_{0}$ and $\beta_{1}$ such that

$$
\begin{aligned}
& A_{0}(n ; c) \sim C_{0} n^{\beta_{0}}, \\
& B_{0}(n ; c) \sim C_{1} n^{\beta_{1}},
\end{aligned}
$$

for some $C_{0}$ and $C_{1}$.

However, we can describe the asymptotic behavior of our coefficients functions in the following way:

Proposition 3.1. Let $A_{0}(n ; c), A_{1}(n ; c), B_{0}(n ; c)$ and $B_{1}(n ; c)$ the functions defined by (30). Then, we have

$$
\begin{array}{ll}
A_{1}(n ; c) \sim 1, \quad \lim _{n \rightarrow \infty} n^{\beta} A_{0}(n ; c) & \begin{cases}0 & \text { if } \beta<\frac{1}{2}, \\
\nexists & \text { if } \beta \geqslant \frac{1}{2},\end{cases} \\
B_{1}(n ; c) \sim n, \quad \lim _{n \rightarrow \infty} n^{\beta} B_{0}(n ; c) & \begin{cases}0 & \text { if } \beta<\frac{1}{2}, \\
\nexists & \text { if } \beta \geqslant \frac{1}{2} .\end{cases}
\end{array}
$$

Proof. The asymptotic behavior of $A_{0}(n ; c)$ and $B_{0}(n ; c)$ is an immediate consequence of the estimates in (33).

In order to obtain the asymptotics for $A_{1}(n ; c)$ and $B_{1}(n ; c)$, we joint up the terms

$$
\begin{aligned}
& \cos \varphi_{n 1}^{\alpha}(c) \cos \varphi_{n}^{\alpha}(c)+\cos \varphi_{n 2}^{\alpha+1}(x) \cos \varphi_{n 1}^{\alpha+1}(c) \quad \cos \left(2 \sqrt{ } c(n \quad 1)+\sqrt{ } c n+\sqrt{ } c\left(\begin{array}{llll}
n & 2) & \alpha \pi & \pi
\end{array}\right)\right. \\
& \left.\left.\times \cos \left(\begin{array}{lll}
\sqrt{ } c n & \sqrt{ } c(n & 2
\end{array}\right)+\frac{\pi}{2}\right)+\frac{1}{2} \cos \left(\begin{array}{lll}
2 \sqrt{ } c(n & 1
\end{array}\right) \quad 2 \sqrt{ } c n\right) \\
& +\frac{1}{2} \cos \left(2 \sqrt{ } c\left(\begin{array}{ll}
n & 2
\end{array}\right) \quad 2 \sqrt{ } c\left(\begin{array}{ll}
n & 1
\end{array}\right)\right)
\end{aligned}
$$

and

$$
\cos \varphi_{n}^{\alpha}(c) \cos \varphi_{n}^{\alpha}(c)+\cos \varphi_{n}^{\alpha+1}(c) \cos \varphi_{n}^{\alpha+1}(c) \quad \cos \left(2 \sqrt{ } c n+2 \sqrt{ } c\left(\begin{array}{llll}
n & 1) & \alpha \pi & \pi
\end{array}\right) \cos \left(\begin{array}{lll}
2 \sqrt{ } c n & 2 \sqrt{ } c(n & 1
\end{array}\right)+\frac{\pi}{2}\right)+1 .
$$

Taking into account that the previous expressions tend to 1 as $n$ tends to infinity, we obtain the desired result.

We can now formulate our main result.

Theorem 3.1. The outer relative asymptotics for Laguerre Sobolev type polynomials $\widehat{S}_{n}^{M, N}(x)$, orthogonal with respect to the discrete Sobolev inner product (2), is

$$
\lim _{n \rightarrow \infty} \frac{\widehat{S}_{n}^{M, N}(x)}{\widehat{L}_{n}^{\alpha}(x)} \quad 1,
$$

uniformly on compact subsets of $\mathbb{C} \backslash \mathbb{R}_{+}$.

Proof. Replacing (29) in (28)

$$
\frac{\widehat{S}_{n}^{M, N}(x)}{\widehat{L}_{n}^{\alpha}(x)} \quad\left\{1+\frac{A_{1}(n ; c)}{(x \quad c)}+\frac{A_{0}(n ; c)}{(x \quad c)^{2}}\right\}+\left\{\frac{B_{1}(n ; c)}{(x \quad c)}+\frac{B_{0}(n ; c)}{(x \quad c)^{2}}\right\} \frac{\widehat{L}_{n}^{\alpha}(x)}{\widehat{L}_{n}^{\alpha}(x)},
$$


From the Perron's formula (11) (for more details we refer the reader to [6]) we get

$$
\frac{L_{n}^{(\alpha)}(x)}{L_{n}^{(\alpha)}(x)} \quad 1 \quad \frac{\sqrt{ } x}{\sqrt{ } n}+\mathcal{O}\left(n^{1}\right)
$$

For monic polynomials (10) the above relation becomes

$$
\frac{\widehat{L}_{n 1}^{\alpha}(x)}{\widehat{L}_{n}^{\alpha}(x)} \quad \frac{1}{n}\left(1 \quad \frac{\sqrt{ } x}{\sqrt{ } n}+\mathcal{O}\left(n^{1}\right)\right) .
$$

By using (35) we can rewrite (34) as

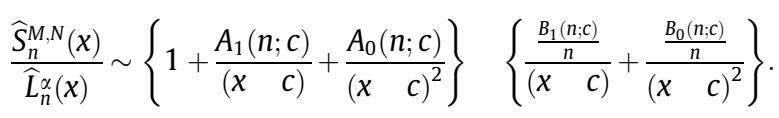

Then, in order to conclude our proof, we only need to check that

$$
\begin{aligned}
& \lim _{n \rightarrow \infty}\left(A_{1}(n ; c) \frac{B_{1}(n ; c)}{n}\right) \quad 0, \\
& \lim _{n \rightarrow \infty}\left(A_{0}(n ; c) \frac{B_{0}(n ; c)}{n}\right) \quad 0 .
\end{aligned}
$$

By applying Proposition 3.1, we obtain (36). From (33), we get

$$
\begin{aligned}
& A_{0}(n ; c) \quad \frac{B_{0}(n ; c)}{n} \sim \frac{1}{M c \sigma^{\alpha}(c) \sigma^{\alpha+3}(c)} n^{1}\left(\cos \varphi_{n}^{\alpha}(c) \cos \varphi_{n 1}^{\alpha+1}(c) \quad \cos \varphi_{n}^{\alpha}(c) \cos \varphi_{n}^{\alpha+1}(c)\right) \\
& c^{1 / 2} n^{1 / 2}\left(\cos \varphi_{n}^{\alpha}{ }_{1}(c) \cos \varphi_{n}^{\alpha+1}(c) \quad \cos \varphi_{n}^{\alpha}(c) \cos \varphi_{n}^{\alpha+1}(c)\right) 2 n^{1} \\
& \times\left(\cos \varphi_{n}^{\alpha}{ }_{1}(c) \cos \varphi_{n}^{\alpha}(c) \quad \cos \varphi_{n}^{\alpha}(c) \cos \varphi_{n}^{\alpha}(c)\right)
\end{aligned}
$$

Since this expression tend to zero as $n$ tends to infinity, then (37) hold.

\section{The five-term recurrence relation}

This section is focused on the five term recurrence relation that the sequence of discrete Laguerre Sobolev orthogonal polynomials $\left\{\widehat{S}_{n}^{M, N}(x)\right\}_{n \geqslant 0}$ satisfies. Next, we will estimate the coefficients of such a recurrence relation for $n$ large enough and $c \in \mathbb{R}_{+}$. To this end, we will use the remarkable fact, which is a straightforward consequence of (2), that the multiplica tion operator by $\left(\begin{array}{ll}x & c\end{array}\right)^{2}$ is a symmetric operator with respect to such a discrete Sobolev inner product. Indeed, for any $f(x), g(x) \in \mathbb{P}$

$$
\left\langle\left(\begin{array}{ll}
x & c
\end{array}\right)^{2} f(x), g(x)\right\rangle_{S} \quad\left\langle f(x),\left(\begin{array}{ll}
x & c
\end{array}\right)^{2} g(x)\right\rangle_{S} .
$$

Notice that

$$
\left\langle(x \quad c)^{2} f(x), g(x)\right\rangle_{S} \quad\langle f(x), g(x)\rangle_{[2]} .
$$

An equivalent formulation of (39) is

$$
\left\langle\left(\begin{array}{ll}
x & c
\end{array}\right)^{2} f(x), g(x)\right\rangle_{S} \quad\left\langle\left(\begin{array}{ll}
x & c
\end{array}\right)^{2} f(x), g(x)\right\rangle_{\alpha} .
$$

We will need some preliminary results that will be stated as Lemmas 4.1 and 4.2.

Lemma 4.1. For every $n \geqslant 1$ and initial conditions $\widehat{L}_{-1}^{\alpha}(x) \quad 0, \widehat{L}_{0}^{\alpha}(x) \quad 1, \widehat{L}_{1}^{\alpha}(x) \quad x \quad(\alpha+1)$, the connection formula (28) reads as

$$
\left(\begin{array}{lll}
x & c
\end{array}\right)^{2} \widehat{S}_{n}^{M, N}(x) \quad \widehat{L}_{n+2}^{\alpha}(x)+\tilde{b}_{n} \widehat{L}_{n+1}^{\alpha}(x)+\tilde{c}_{n} \widehat{L}_{n}^{\alpha}(x)+\tilde{d}_{n} \widehat{L}_{n 1}^{\alpha}(x)+\tilde{e}_{n} \widehat{L}_{n 2}^{\alpha}(x),
$$

where

$$
\begin{aligned}
& \tilde{b}_{n} \quad \beta_{n+1}+\beta_{n} \quad 2 c+A_{1}(n ; c) \sim 4 n,
\end{aligned}
$$

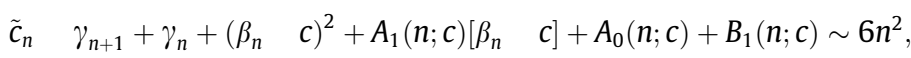

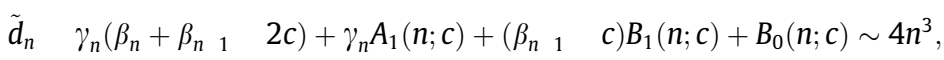

$$
\begin{aligned}
& \tilde{e}_{n} \quad \gamma_{n} \gamma_{n 1}+\gamma_{n 1} B_{1}(n ; c) \sim n^{4} \text {. }
\end{aligned}
$$


Proof. We begin with the expression

$$
\left(\begin{array}{lll}
x & c
\end{array}\right)^{2} \widehat{L}_{n}^{\alpha}(x) \quad \widehat{L}_{n+2}^{\alpha}(x)+b_{n} \widehat{L}_{n+1}^{\alpha}(x)+c_{n} \widehat{L}_{n}^{\alpha}(x)+d_{n} \widehat{L}_{n 1}^{\alpha}(x)+e_{n} \widehat{L}_{n 2}^{\alpha}(x),
$$

where

$b_{n} \quad \beta_{n+1}+\beta_{n} \quad 2 c \sim 4 n, \quad c_{n} \quad \gamma_{n+1}+\gamma_{n}+\left(\begin{array}{lllllll}\beta_{n} & c\end{array}\right)^{2} \sim 6 n^{2}, \quad d_{n} \quad \gamma_{n}\left(\beta_{n}+\beta_{n} 1 \quad 2 c\right) \sim 4 n^{3}, \quad e_{n} \quad \gamma_{n} \gamma_{n 1} \sim n^{4}$, according to (4) and the definition of $\beta_{n}$ and $\gamma_{n}$ in (4).

From the expression of $A(n ; x)$ in (29), the next step is to expand the polynomial $\left[A_{1}(n ; x)(x \quad c)+A_{0}(n ; x)\right] \widehat{L}_{n}^{\alpha}(x)$ in terms of $\left\{\widehat{L}_{n}^{\alpha}\right\}_{n \geqslant 0}$. Indeed, from (4)

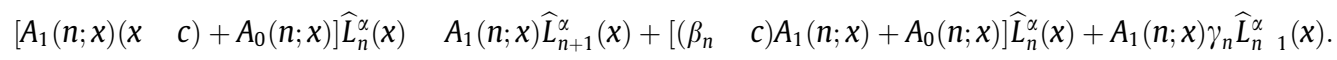

Adding these coefficients to those of (41), we obtain

$$
A(n ; x) \widehat{L}_{n}^{\alpha}(x) \quad \widehat{L}_{n+2}^{\alpha}(x)+b_{n} \widehat{L}_{n+1}^{\alpha}(x)+c_{n} \widehat{L}_{n}^{\alpha}(x)+d_{n} \widehat{L}_{n 1}^{\alpha}(x)+e_{n} \widehat{L}_{n 2}^{\alpha}(x)
$$

with

$$
b_{n} \quad b_{n}+A_{1}(n ; c) \sim 4 n, \quad c_{n} \quad c_{n}+A_{1}(n ; c)\left(\beta_{n} \quad c\right)+A_{0}(n ; c) \sim 6 n^{2}, \quad d_{n} \quad d_{n}+\gamma_{n} A_{1}(n ; c) \sim 4 n^{3}, \quad e_{n} \quad e_{n} \sim n^{4},
$$

where we have used Proposition 3.1. In a similar way, for $B(n ; x)$ in (29) we get

$$
B(n ; x) \widehat{L}_{n}^{\alpha}{ }_{1}(x) \quad \breve{c}_{n} \widehat{L}_{n}^{\alpha}(x)+\breve{d}_{n} \widehat{L}_{n 1}^{\alpha}(x)+\breve{e}_{n} \widehat{L}_{n 2}^{\alpha}(x),
$$

where

$$
\begin{array}{ll}
\breve{c}_{n} & B_{1}(n ; c) \sim n, \\
\breve{d}_{n} & \left(\beta_{n} 1 \quad c\right) B_{1}(n ; c)+B_{0}(n ; c) \sim 2 n^{2}, \\
\breve{e}_{n} & \gamma_{n}{ }_{1} B_{1}(n ; c) \sim n^{3} .
\end{array}
$$

As a conclusion,

$$
\left(\begin{array}{lll}
x & c
\end{array}\right)^{2} \widehat{S}_{n}^{M, N}(x) \quad A(n ; x) \widehat{L}_{n}^{\alpha}(x)+B(n ; x) \widehat{L}_{n 1}^{\alpha}(x) \quad \widehat{L}_{n+2}^{\alpha}(x)+b_{n} \widehat{L}_{n+1}^{\alpha}(x)+\left(c_{n}+\breve{c}_{n}\right) \widehat{L}_{n}^{\alpha}(x)+\left(d_{n}+\breve{d}_{n}\right) \widehat{L}_{n 1}^{\alpha}(x)+\left(e_{n}+\breve{e}_{n}\right) \widehat{L}_{n 2}^{\alpha}(x) .
$$

This completes the proof.

Lemma 4.2. For every $\alpha>1, n \geqslant 1$, and $c \in \mathbb{R}_{+}$the norm of the Laguerre Sobolev type polynomials $\widehat{S}_{n}^{M, N}$, orthogonal with respect to (2) is

$$
\left\|\widehat{S}_{n}^{M, N}\right\|_{S}^{2} \quad\left\|\widehat{L}_{n}^{\alpha}\right\|_{\alpha}^{2}+B_{1}(n ; c)\left\|\widehat{L}_{n}^{\alpha}\right\|_{\alpha}^{2} \sim \Gamma(n+1) \Gamma(n+\alpha+1) .
$$

where $B_{1}(n ; c)$ is the polynomial coefficient defined in (30).

Proof. First, we notice that

$$
\left\|\widehat{S}_{n}^{M, N}\right\|_{S}^{2} \quad\left\langle\widehat{S}_{n}^{M, N}(x),\left(\begin{array}{ll}
x & c
\end{array}\right)^{2} \widehat{\Pi}_{n 2}(x)\right\rangle_{S},
$$

for every monic polynomial $\widehat{\Pi}_{n 2}$ of degree $n \quad 2$. From (40)

$$
\left\langle\widehat{S}_{n}^{M, N}(x),\left(\begin{array}{lll}
x & c
\end{array}\right)^{2} \widehat{\Pi}_{n_{2}}(x)\right\rangle_{S} \quad\left\langle\left(\begin{array}{ll}
x & c
\end{array}\right)^{2} \widehat{S}_{n}^{M, N}(x), \widehat{\Pi}_{n_{2}}(x)\right\rangle_{S} \quad\left\langle\left(\begin{array}{ll}
x & c
\end{array}\right)^{2} \widehat{S}_{n}^{M, N}(x), \widehat{\Pi}_{n 2}(x)\right\rangle_{\alpha} .
$$

Next we use the connection formula (28). Taking into account that $A(n ; x)$ is a monic quadratic polynomial and $B(n ; x)$ is a linear polynomial with leading coefficient $B_{1}(n ; c)$,

$$
\begin{aligned}
\left\|\widehat{S}_{n}^{M, N}\right\|_{S}^{2} & \left\langle(x \quad c)^{2} \widehat{S}_{n}^{M, N}(x), \widehat{\Pi}_{n 2}(x)\right\rangle_{\alpha} \quad\left\langle A(n ; x) \widehat{L}_{n}^{\alpha}(x), \widehat{\Pi}_{n 2}(x)\right\rangle_{\alpha}+\left\langle B(n ; x) \widehat{L}_{n 1}^{\alpha}(x), \widehat{\Pi}_{n 2}(x)\right\rangle_{\alpha} \\
& \left\langle\widehat{L}_{n}^{\alpha}(x), x^{n}\right\rangle_{\alpha}+B_{1}(n ; c)\left\langle\widehat{L}_{n 1}^{\alpha}(x), x^{n}{ }^{1}\right\rangle_{\alpha} .
\end{aligned}
$$

The first term in the above expression is the norm of the monic Laguerre polynomial of degree $n$ and the second one is the norm of the Laguerre polynomial of degree $n \quad 1$ times $B_{1}(n ; c)$, which is given in (30). This means

$$
\left\|\widehat{S}_{n}^{M, N}\right\|_{s}^{2} \quad\left\|\widehat{L}_{n}^{\alpha}\right\|_{\alpha}^{2}+B_{1}(n ; c)\left\|\widehat{L}_{n}^{\alpha}\right\|_{\alpha}^{2} .
$$

Using the estimates (5) and Proposition 3.1, we obtain

$$
\left\|\widehat{S}_{n}^{M, N}\right\|_{S}^{2} \sim \Gamma(n+1) \Gamma(n+\alpha+1),
$$


which completes the proof.

We are ready to find the five term recurrence relation satisfied by $\widehat{S}_{n}^{M, N}(x)$, and the asymptotic behavior of the correspond ing coefficients. Next, we will focus our attention on its proof.

Let consider the Fourier expansion of $(x \quad c)^{2} \widehat{S}_{n}^{M, N}(x)$ in terms of $\left\{\widehat{S}_{n}^{M, N}(x)\right\}_{n \geqslant 0}$

$$
\left(\begin{array}{ll}
x & c
\end{array}\right)^{2} \widehat{S}_{n}^{M, N}(x) \quad \sum_{k 0}^{n+2} \lambda_{n, k} \widehat{S}_{k}^{M, N}(x)
$$

where

$$
\lambda_{n, k} \quad \frac{\left\langle(x \quad c)^{2} \widehat{S}_{n}^{M, N}(x), \widehat{S}_{k}^{M, N}(x)\right\rangle_{S}}{\left\|\widehat{S}_{k}^{M, N}\right\|_{S}^{2}}, \quad k \quad 0, \ldots, n+2 .
$$

Thus, $\lambda_{n, k} \quad 0$ for $k \quad 0, \ldots, n \quad 3$. We are dealing with monic polynomials, so the leading coefficient $\lambda_{n, n+2} \quad 1$. To obtain $\lambda_{n, n+1}$, we use the connection formula (28), with coefficients $A(n ; x)$ and $B(n ; x)$ as in (29). Thus,

$$
\begin{aligned}
\lambda_{n, n+1} & \frac{1}{\left\|\widehat{S}_{n+1}^{M, N}\right\|_{S}^{2}}\left\langle A(n ; x) \widehat{L}_{n}^{\alpha}(x), \widehat{S}_{n+1}^{M, N}(x)\right\rangle_{S}+\frac{1}{\left\|\widehat{S}_{n+1}^{M, N}\right\|_{S}^{2}}\left\langle B(n ; x) \widehat{L}_{n}^{\alpha}{ }_{1}(x), \widehat{S}_{n+1}^{M, N}(x)\right\rangle_{S} \\
& \frac{1}{\left\|\widehat{S}_{n+1}^{M, N}\right\|_{S}^{2}}\left\langle(x \quad c)^{2} \widehat{L}_{n}^{\alpha}(x), \widehat{S}_{n+1}^{M, N}(x)\right\rangle_{S}+A_{1}(n ; c) .
\end{aligned}
$$

Let us study the discrete Sobolev inner product $\left\langle\left(\begin{array}{ll}x & c\end{array}\right)^{2} \widehat{L}_{n}^{\alpha}(x), \widehat{S}_{n+1}^{M, N}(x)\right\rangle_{S}$ above. Applying (38), (40), (5) and Lemma 4.1, we obtain

$$
\left\langle\left(\begin{array}{ll}
x & c
\end{array}\right)^{2} \widehat{L}_{n}^{\alpha}(x), \widehat{S}_{n+1}^{M, N}(x)\right\rangle_{S} \quad\left\langle\widehat{L}_{n}^{\alpha}(x),\left(\begin{array}{ll}
x & c
\end{array}\right)^{2} \widehat{S}_{n+1}^{M, N}(x)\right\rangle_{\alpha} \quad \tilde{d}_{n+1}\left\|\widehat{L}_{n}^{\alpha}\right\|_{\alpha}^{2} .
$$

From (23), Lemma 4.2 and Proposition 3.1

$$
\lambda_{n, n+1} \frac{\tilde{d}_{n+1}\left\|\widehat{L}_{n}^{\alpha}\right\|_{\alpha}^{2}+A_{1}(n ; c)}{\left\|\widehat{S}_{n+1}^{M, N}\right\|_{S}^{2}} \sim 4 n .
$$

In order to compute $\lambda_{n, n}$, from (28) and (29) we get

$$
\lambda_{n, n} \frac{\left\langle(x \quad c)^{2} \widehat{L}_{n}^{\alpha}(x), \widehat{S}_{n}^{M, N}(x)\right\rangle_{S}}{\left\|\widehat{S}_{n}^{M, N}\right\|_{S}^{2}}+A_{1}(n ; c) \frac{\left\langle(x \quad c) \widehat{L}_{n}^{\alpha}(x), \widehat{S}_{n}^{M, N}(x)\right\rangle_{S}}{\left\|\widehat{S}_{n}^{M, N}\right\|_{S}^{2}}+A_{0}(n ; c)+B_{1}(n ; c) .
$$

But, according to (38) and (40) and Lemma 4.1, the first term is

$$
\frac{\left\langle(x \quad c)^{2} \widehat{L}_{n}^{\alpha}(x), \widehat{S}_{n}^{M, N}(x)\right\rangle_{S}}{\left\|\widehat{S}_{n}^{M, N}\right\|_{S}^{2}} \quad \tilde{c}_{n} \frac{\left\|\widehat{L}_{n}^{\alpha}\right\|_{\alpha}^{2}}{\left\|\widehat{S}_{n}^{M, N}\right\|_{S}^{2}}
$$

After some algebraic manipulations, from (4) we get

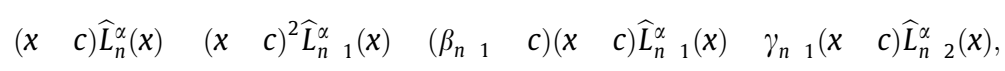

Using this expression, we obtain

$$
\frac{\left\langle(x \quad c) \widehat{L}_{n}^{\alpha}(x), \widehat{S}_{n}^{M, N}(x)\right\rangle_{S}}{\left\|\widehat{S}_{n}^{M, N}\right\|_{S}^{2}} \frac{\left\langle\widehat{L}_{n}^{\alpha}{ }_{1}(x),(x \quad c)^{2} \widehat{S}_{n}^{M, N}(x)\right\rangle_{\alpha}}{\left\|\widehat{S}_{n}^{M, N}\right\|_{S}^{2}} \quad\left(\begin{array}{lllll}
\beta_{n} & 1 & c
\end{array}\right) \quad \tilde{d}_{n} \frac{\left\|\widehat{L}_{n}^{\alpha}\right\|_{\alpha}^{2}}{\left\|\widehat{S}_{n}^{M, N}\right\|_{S}^{2}} \quad\left(\begin{array}{lll}
\beta_{n} & 1 & c
\end{array}\right) .
$$

As a consequence, we get

$$
\lambda_{n, n} \frac{\tilde{c}_{n}\left\|\widehat{L}_{n}^{\alpha}\right\|_{\alpha}^{2}+\tilde{d}_{n}\left\|\widehat{L}_{n}^{\alpha}\right\|_{\alpha}^{2}+\left(\beta_{n 1} \quad c\right)+A_{0}(n ; c)+B_{1}(n ; c)}{\left\|\widehat{S}_{n}^{M, N}\right\|_{S}^{2}} \sim 6 n^{2} .
$$

A similar analysis yields

$$
\begin{array}{ll}
\lambda_{n, n} 1 & \frac{\tilde{d}_{n}\left\|\widehat{L}_{n}^{\alpha} 1\right\|_{\alpha}^{2}+A_{1}(n \quad 1 ; c)\left\|\widehat{S}_{n}^{M, N}\right\|_{S}^{2}}{\left\|\widehat{S}_{n, 1}^{M, N}\right\|_{S}^{2}} \sim 4 n^{3}, \\
\lambda_{n, n} 2 & \frac{\left\|\widehat{S}_{n}^{M, N}\right\|_{S}^{2}}{\left\|\widehat{S}_{n}^{M, N}\right\|_{S}^{2}} \sim n^{4} .
\end{array}
$$

We can summarize the results of this Section in the following theorem. 
Theorem 4.1 (Five term recurrence relation). For every $n \geqslant 1, \alpha>1$ and $c \in \mathbb{R}_{+}$, the monic Laguerre Sobolev type polynomials $\left\{\widehat{S}_{n}{ }^{M, N}\right\}_{n \geqslant 0}$, orthogonal with respect to (2) satisfy the following five term recurrence relation



with

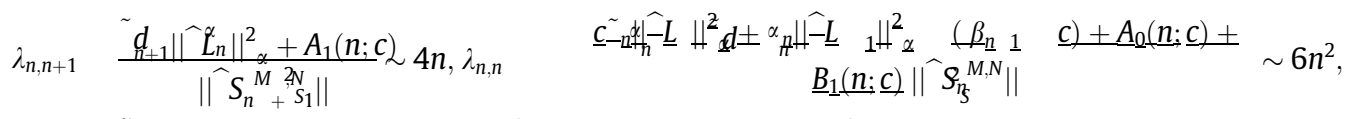



\section{Acknowledgements}

The authors thank the reviewers for their careful revision of the manuscript. Their helpful comments and suggestions contributed to improve substantially style and presentation of the manuscript.

\section{References}

[1] M. Alfaro, F. Marcellán, M.L. Rezola, A. Ronveaux, Sobolev-type orthogonal polynomials: the non-diagonal case, J. Approx. Theory 83 (1995) $266-287$.

[2] I. Alvarez Rocha, L. Salto, Asymptotics of polynomials orthogonal with respect to a discrete-complex Sobolev inner product, J. Comput. Appl. Math. 178 (2005) 1-19.

[3] I. Baratchart, A. Martínez-Finkelshtein, D. Jiménez, D.S. Lubinsky, H.N. Mhaskar, I. Pritsker, M. Putinar, M. Stylianopoulus, V. Totik, P. Varju, Y. Xu, Open problems in constructive function theory, Electron. Trans. Numer. Anal. 25 (2006) 511-525.

[4] A. Branquinho, A. Foulquié, F. Marcellán, Asymptotic behavior of Sobolev type orthogonal polynomials on a rectifiable Jordan curve or arc, Constr. Approx. 18 (2002) 161-182.

[5] T.S. Chihara, An Introduction to Orthogonal Polynomials, Mathematics and its Applications Series, Gordon and Breach, New York, 1978.

[6] A. Deaño, E.J. Huertas, F. Marcellán, Strong and ratio asymptotics for Laguerre polynomials revisited, J. Math. Anal. Appl. 403 (2013) 477-486.

[7] H. Dueñas, F. Marcellán, Asymptotic behavior of Laguerre-Sobolev type orthogonal polynomials: a nondiagonal case, J. Comput. Appl. Math. 235 (2010) 998-1007.

[8] H. Dueñas, F. Marcellán, The Laguerre-Sobolev-type orthogonal polynomials. Holonomic equation and electrostatic interpretation, Rocky Mt. J. Math. 41 (2011) 95-131.

[9] H. Dueñas, E.J. Huertas, F. Marcellán, Analytic properties of Laguerre-type orthogonal polynomials, Integral Transforms Spec. Funct. 22 (2011) 107-122.

[10] H. Dueñas, E.J. Huertas, F. Marcellán, Asymptotic properties of Laguerre-Sobolev type orthogonal polynomials, Numer. Algorithms 60 (2012) 51-73.

[11] W.D. Evans, L.L. Littlejohn, F. Marcellán, C. Markett, A. Ronveaux, On recurrence relations for Sobolev orthogonal polynomials, SIAM J. Math. Anal. 26 (1995) 446-467.

[12] B.Xh. Fejzullahu, R.Xh. Zejnullahu, Orthogonal polynomials with respect to the Laguerre measure perturbed by the canonical transformations, Integral Transforms Spec. Funct. 21 (2010) 569-580.

[13] A. Foulquié, F. Marcellán, K. Pan, Asymptotic behavior of Sobolev-type orthogonal polynomials on the unit circle, J. Approx. Theory 100 (1999) 345363.

[14] E.J. Huertas, Analytic properties of Krall-type and Sobolev-type orthogonal polynomials (Doctoral Dissertation), Universidad Carlos III de Madrid, 2012.

[15] M.E.H. Ismail, Classical and Quantum Orthogonal Polynomial in One Variable, Encyclopedia of Mathematics and its Applications, vol. 98, Cambridge University Press, Cambridge, 2005.

[16] G. López Lagomasino, Relative asymptotics for polynomials orthogonal on the real axis, Mat. Sb. (N.S.) 137(179) (1988), 500-525, 57. (English translation (1990): Math. USSR-Sb. 65, 505-529)

[17] G. López Lagomasino, F. Marcellán, W. Van Assche, Relative symptotics for polynomials orthogonal with respect to a discrete Sobolev inner product, Constr. Approx. 11 (1995) 107-137.

[18] F. Marcellán, A. Branquinho, J.C. Petronilho, Classical orthogonal polynomials: a functional approach, Acta Appl. Math. 34 (1994) $283-303$.

[19] F. Marcellán, J.J. Moreno-Balcázar, Asymptotics and zeros of Sobolev orthogonal polynomials on unbounded supports, Acta Appl. Math. 94 (2006) 163192.

[20] F. Marcellán, B.P. Osilenker, I.A. Rocha, On Fourier series of Jacobi-Sobolev orthogonal polynomials, J. Inequal. Appl. 7 (2002) $673-699$.

[21] F. Marcellán, B.P. Osilenker, I.A. Rocha, On Fourier series of a discrete Jacobi-Sobolev inner product, J. Approx. Theory 117 (2002) 1-22.

[22] F. Marcellán, M.F. Pérez-Valero, Y. Quintana, A. Urieles, Recurrence relations and outer relative asymptotics of orthogonal polynomials with respect to a discrete Sobolev type inner product, Bull. Math. Sci. (2013), http://dx.doi.org/10.1007/s13373-013-0047-X.

[23] F. Marcellán, Y. Quintana, A. Urieles, On $W^{1, p}$-convergence of Fourier-Sobolev expansions, J. Math. Anal. Appl. 398 (2013) 594-599.

[24] F. Marcellán, Y. Quintana, A. Urieles, On the Pollard decomposition method applied to some Jacobi-Sobolev expansions, Turk. J. Math. 37 (2013) 934948.

[25] F. Marcellán, A. Ronveaux, On a class of polynomials orthogonal with respect to a discrete Sobolev inner product, Indag. Math. (N.S.) 1 (4) (1990) 451464.

[26] F. Marcellán, R.Xh. Zejnullahu, B.Xh. Fejzullahu, E.J. Huertas, On orthogonal polynomials with respect to certain discrete Sobolev inner product, Pacific J. Math. 257 (2012) 167-188.

[27] F. Marcellán, W. Van Assche, Relative asymptotics for orthogonal polynomials with a Sobolev inner product, J. Approx. Theory 72 (1993) 193-209.

[28] G. López Lagomasino, H. Pijeira, Zero location and $n$th root asymptotics of Sobolev orthogonal polynomials, J. Approx. Theory 99 (1999) 30-43.

[29] G. López Lagomasino, I. Pérez Izquierdo, H. Pijeira, Asymptotic of extremal polynomials in the complex plane, J. Approx. Theory 137 (2005) $226-237$.

[30] P. Nevai, Orthogonal Polynomials, Memoirs of the American Mathematical Society, vol. 213, American Mathematical Society Providence, RI, 1979.[31]

A.F. Nikiforov, V.B. Uvarov, Special Functions of Mathematical Physics: An Unified Approach, Birkhauser Verlag, Basel, 1988.

[32] B.P. Osilenker, Fourier Series in Orthogonal Polynomials, World Scientific, Singapore, 1999.

[33] A. Portilla, J.M. Rodríguez, E. Tourís, The multiplication operator, zero location and asymptotic for non-diagonal Sobolev norms, Acta Appl. Math. 111 (2010) 205-218.

[34] A. Portilla, Y. Quintana, J.M. Rodríguez, E. Tourís, Zero location and asymptotic behavior for extremal polynomials with non-diagonal Sobolev norms, J. Approx. Theory 162 (2010) 2225-2242.

[35] A. Portilla, Y. Quintana, J.M. Rodríguez, E. Tourís, Concerning asymptotic behavior for extremal polynomials associated to non-diagonal Sobolev norms, J. Funct. Spaces Appl. (2013) 1-11 (article ID 628031). 
[36] E.A. Rakhmanov, On the asymptotics of the ratio of orthogonal polynomials, Mat. Sb. 103 (145) (1977) 271-291 (English translation, Math. USSR-Sb. 32 (1977) 199-213).

[37] E.A. Rakhmanov, On the asymptotics of the ratio of orthogonal polynomials II, Mat. Sb. 118 (160) (1982) 271-291 (English translation (1983): Math. USSR-Sb. 46, 105-117).

[38] E.A. Rakhmanov, On the asymptotics of polynomials orthogonal on the circle with weights not satisfying Szeg"o's condition, Mat. Sb. 118 (172)

(1986)51-169 (English translation (1987): Math. USSR-Sb. 58, 149-167).

[39] I.A. Rocha, F. Marcellán, L. Salto, Relative asymptotics and Fourier series of orthogonal polynomials with a discrete Sobolev inner product, J. Approx. Theory 121 (2003) 336-356.

[40] H. Stahl, V. Totik, General Orthogonal Polynomials, Cambridge University Press, Cambridge, 1992.

[41] G. Szeg"o, Orthogonal Polynomials, Colloquim Publications American Mathematical Society, fourth ed., 23, American Mathematical Society Providence, RI, 1975. 